\title{
Hybrid adaptive observers for locally Lipschitz systems with application to mechanical oscillators
}

\author{
Alexey A. Bobtsov, Member, IEEE, Denis V. Efimov, Member, IEEE, Anton Pyrkin, Member, IEEE
}

\begin{abstract}
Adaptive observer design procedure is proposed for nonlinear locally Lipschitz systems. Possible presence of disturbances is taken into account. The solution is based on logic-based control approach applicable to nonlinear systems with bounded solutions. Efficacy of the proposed observer is demonstrated by computer simulation for a mechanical oscillating system.
\end{abstract}

\section{INTRODUCTION}

$\mathrm{T}_{\mathrm{b}}^{\mathrm{h}}$ HE observers design problem for nonlinear systems has been an area of intensive research during the last two decades. There exist a lot of solutions in the area dealing with diverse forms of systems models. Among them it is necessary to mention high gain techniques [4], [9], [15]; sliding mode observers [7], [8], [31], [37]; nonlinear coordinate changes [5], [19], [20], [21]; numerical techniques [23]; approaches dealing with smooth [14], [34] and nonsmooth output functions [16]. Adaptive observers are designed for systems with parametric uncertainties [6], [12], [33], [35], [38]. Observers design procedures find their applications not only in areas of control under partial measurements [15], [40], but also for fault detection [3], [13], [33], [36] systems synchronization [22] and secured data transmission and encoding [12], [24].

The class of Lipschitz nonlinear systems has seen much attention:

$$
\dot{\mathbf{x}}=\mathbf{A} \mathbf{x}+\varphi(\mathbf{y})+\mathbf{B} \mathbf{f}(\mathbf{x}, \mathbf{d}), \mathbf{y}=\mathbf{C} \mathbf{x},
$$

where $\mathbf{x} \in R^{n}$ is state vector; $\mathbf{d} \in R^{m}$ is disturbing input; $\mathbf{y} \in R^{p}$ is available for measurements output and functions $\boldsymbol{\varphi}: R^{p} \rightarrow R^{n}$ and $\mathbf{f}: R^{n+m} \rightarrow R^{p}$ are Lipschitz continuous (function f globally); constant matrices A, B, C have appropriate dimensions. An advance of this class of systems consists in fact, that almost all nonlinear systems of the form

$$
\dot{\mathbf{x}}=\mathbf{F}(\mathbf{x}, \mathbf{d}), \mathbf{y}=\mathbf{C} \mathbf{x},
$$

where $\mathbf{F}: R^{n+m} \rightarrow R^{n}$ is locally Lipschitz continuous, can

The first and the last authors are with Department of Control Systems and Informatics, Saint-Petersburg State University of Information Technologies Mechanics and Optics, Kronverksky av. 49, St.Petersburg, 197101, Russia, bobtsov@mail.ru. The second author is with CCS Lab., Institute for Problems of Mechanical Engineering, Bolshoi av., 61, V.O., St. Petersburg, 199178, Russia and Systems and Control @ Systems and Modeling, Department of Electrical Engineering and Computer Science, B28 Université de Liège, B-4000 Liege Sart-Tilman, Belgium.

This paper presents research results of the Belgian Network DYSCO, funded by the Interuniversity Attraction Poles Programme, initiated by the Belgian State, Science Policy Office. The scientific responsibility rests with its author(s).and it is partly supported by RFBR grant 08-01-00775. be reduced to (1) at least locally. Tacking in mind possible parametric uncertainties presence, the equations of system (1) can be generalized as follows:

$$
\dot{\mathbf{x}}=\mathbf{A} \mathbf{x}+\boldsymbol{\varphi}(\mathbf{y})+\mathbf{B}[\mathbf{f}(\mathbf{x}, \mathbf{d})+\mathbf{G}(\mathbf{x}) \boldsymbol{\theta}], \mathbf{y}=\mathbf{C} \mathbf{x},
$$

where $\boldsymbol{\theta} \in R^{p}$ is constant vector of unknown parameters and $\mathbf{G}: R^{n} \rightarrow R^{p \times p}$ is continuous and locally Lipschitz matrix function.

Observers design for Lipschitz systems (1) was considered in [13, 27-30, 32]. Robust properties of observers for (1) with respect to disturbing input $\mathbf{d}$ were analyzed in [1], [3], [25]. Adaptive observers design problem for Lipschitz systems was solved in [6], [35]. All these solutions were obtained under assumption on globality of Lipschitz property for function $\mathbf{f}$. Under such assumption applying sufficiently high observer feedback gain it is possible to cancel influence of nonlinearity on observation error dynamics and the problem is solvable via linear systems approach.

Application of sliding mode approach allows one to ensure finite time convergence of a part of observation error to zero [7], [8], [31], [37]. Additionally, equivalent control methodic may help to estimate disturbances levels affecting on the system (that is useful for fault detection). Generically application of sliding mode approach for observation improves quality of transient processes and rate of error convergence.

If system is locally Lipschitz, only local solution is possible applying conventional approaches [28], [30], [32]. In [18], [39] attempts were carried out to find global solutions for special classes of systems (1) (without disturbances) based on tuned observer gains, which are continuously increasing while observation error is converging to zero. Development of this approach for generic case (enlarging class of systems or adding disturbances) meets obstacles dealing with possible unboundedness of observer feedback gains. In this work a solution of the problem is proposed for perturbed system (1), where growing observer gains are updated by discrete algorithm. On each step the new gains are substituted in the observer using logic-based scheme if the previous observer gains fail to satisfy some performance criteria. This prevents infinite growth of the gains in the case of disturbances presence.

This technique is further developed for adaptive observers design for system (2). The main difficulty with adaptive observer design for system (2) applying [12], [38] is dependence of nonlinear functions $\mathbf{f}$ and $\mathbf{G}$ on unmeasured vector $\mathbf{x}$. Utilizing hybrid robust state observer technique 
proposed for system (1) it is possible to derive estimates for vector $\mathbf{x}$. Using these estimates and results of [12], [36], [38] it is possible to design adaptive observer for the system, which identifies values of vector of unknown parameters $\boldsymbol{\theta}$. A closely connected solution for globally Lipschitz systems was presented in [37], where an adaptive observer was designed for extended delayed system (without disturbances), that results to rather high dimension of the observer.

The structure of the paper is as follows. Preliminary results are introduced in section 2. State observer design and adaptive observer design are presented in sections 3 and 4 . Results of computer simulations are discussed in section 5 .

\section{PRELIMINARIES}

The norm of Lebesgue measurable and essentially bounded function $\mathbf{d}: R_{+} \rightarrow R^{m}$ of time $t \geq 0$ will be defined as follows

$$
\|\mathbf{d}\|_{\left[t_{0}, t\right)}=\operatorname{ess} \sup \left\{|\mathbf{d}(t)|, t \in\left[t_{0}, t\right)\right\} .
$$

The set of all such functions with property $\|\mathbf{d}\|_{[0,+\infty)}=\|\mathbf{d}\|<+\infty$ we will further denote as $\mathcal{M}_{R^{m}}$.

Norm $|\mathbf{R}|$ of matrix $\mathbf{R}$ is calculated as square root of its maximum singular value. As usual, continuous function $\sigma: R_{+} \rightarrow R_{+}$belongs to class $K$ if it is strictly increasing and $\sigma(0)=0$; additionally it belongs to class $K_{\infty}$ if it is also radially unbounded; and continuous function $\beta: R_{+} \times R_{+} \rightarrow R_{+}$is from class $\mathcal{K} \mathcal{L}$, if $\beta(\cdot, r) \in \mathcal{K}$ for any fixed $r \in R_{+}$, and $\beta(s, \cdot)$ is strictly decreasing to zero for any fixed $s \in R_{+}$.

If for all initial conditions $\mathbf{x}_{0} \in R^{n}$ and inputs $\mathbf{d} \in \mathcal{M}_{R^{m}}$ the solutions $\mathbf{x}\left(t, \mathbf{x}_{0}, \mathbf{d}\right)$ of the system (1) are defined for all $t \geq 0$, then the systems are called forward complete (necessary and sufficient conditions for a nonlinear system $\dot{\mathbf{x}}=\mathbf{F}(\mathbf{x}, \mathbf{d})$ to be forward complete can be found in [2]).

L e m ma 1 . Let $\mathbf{g}: R^{n} \rightarrow R^{l}$ be continuous and locally Lipschitz function, then there exist functions $\alpha_{1}, \alpha_{2} \in \mathcal{K}$ such, that for all $\mathbf{x} \in R^{n}, \mathbf{z} \in R^{n}$ it holds:

$$
|\mathbf{g}(\mathbf{x})-\mathbf{g}(\mathbf{z})| \leq \alpha_{1}(1+|\mathbf{x}|) \alpha_{2}(1+|\mathbf{z}|)|\mathbf{x}-\mathbf{z}| .
$$

All proofs are omitted due to space limitations. The result of the lemma is somehow related with Lemma 2.5 from [18].

The next property is frequently used in adaptive control theory. Consider a linear dynamical system

$$
\dot{\xi}=\mathbf{A} \boldsymbol{\xi}+\mathbf{B} \mathbf{v}, \boldsymbol{\psi}=\mathbf{C} \boldsymbol{\xi}
$$

with state $\xi \in R^{n}$, input $\boldsymbol{v} \in R^{p}$ and output $\boldsymbol{\psi} \in R^{p}$. Introduce the following notations:

$$
\begin{gathered}
\delta(s)=\operatorname{det}\left(s \mathbf{I}_{n}-\mathbf{A}\right), W(s)=\mathbf{C}\left(s \mathbf{I}_{n}-\mathbf{A}\right)^{-1} \mathbf{B}, \\
\varphi(s)=\delta(s) \operatorname{det}(W(s)), \boldsymbol{\Gamma}=\lim _{s \rightarrow+\infty} s W(s),
\end{gathered}
$$

where $\mathbf{I}_{n}$ is identity matrix of size $n \times n$. Matrix inequalities are understood in sense of quadratic forms. The system is called minimum phase if the polynomial $\varphi(s)$ is Hurwitz (its zeros belong to the open left half-plane). The system is called hyper minimum phase if it is minimum phase and $\Gamma=\Gamma^{T}>0[10]$.

L e m ma 2 [10]. Let $\operatorname{rank}(\mathbf{B})=p$. Then the following properties are equivalent.

$1^{\circ}$. The system is hyper minimum phase.

$2^{\circ}$. There exist matrices $\mathbf{P}=\mathbf{P}^{T}>0$ and $\mathbf{K}$ of sizes $n \times n$ and $n \times p$ respectively such, that

$$
\mathbf{P}(\mathbf{A}-\mathbf{K C})+(\mathbf{A}-\mathbf{K C})^{T} \mathbf{P}<0, \mathbf{P} \mathbf{B}=\mathbf{C}^{T} .
$$

The next property is frequently used in adaptive control systems theory to characterize identification ability of adaptation algorithms.

$\mathrm{D}$ e fin ition 1. The Lebesgue measurable and square integrable matrix function $\mathbf{R}: R_{+} \rightarrow R^{l_{1} \times l_{2}}$ with dimension $l_{1} \times l_{2}$ admits $(\ell, \vartheta)$-persistency of excitation (PE) condition, if there exist strictly positive constants $\ell$ and $\vartheta$ such, that for any $t \geq 0$

$$
\int_{t}^{t+\ell} \mathbf{R}(s) \mathbf{R}(s)^{T} d s \geq \vartheta \mathbf{I}_{l_{1}},
$$

where $\mathbf{I}_{l_{1}}$ denotes identity matrix of dimension $l_{1} \times l_{1}$.

L e m m a 3 [11]. Let us consider time-varying linear $d y$ namical system

$$
\dot{\mathbf{p}}=-\Gamma \mathbf{R}(t) \mathbf{R}(t)^{T} \mathbf{p}+\mathbf{b}(t), t_{0} \geq 0,
$$

where $\mathbf{p} \in R^{l_{1}}, \Gamma$ is a positive definite matrix of dimension $l_{1} \times l_{1}$ and functions $\mathbf{R}: R_{+} \rightarrow R^{l_{1} \times l_{2}}, \mathbf{b}: R_{+} \rightarrow R^{l_{1}}$ are Lebesgue measurable, $\mathbf{b}$ is essentially bounded, function $\mathbf{R}$ is $(\ell, \vartheta)-P E$ for some $\ell>0, \vartheta>0$. Then for any initial condition $\mathbf{p}\left(t_{0}\right) \in R^{l_{1}}$ solutions of the system are defined for all $t \geq t_{0}$ and they admit the estimate $(\gamma>0$ is the smallest eigenvalue of matrix $\Gamma$ )

$$
\begin{aligned}
|\mathbf{p}(t)| & \leq\left|\mathbf{p}\left(t_{0}\right)\right| e^{-0.5 \gamma \vartheta \ell^{-1}\left(t-t_{0}-\ell\right)}+ \\
& +\left(1+2 \vartheta^{-1} \gamma^{-1} e^{-0.5 \vartheta \gamma \ell^{-1}\left(\ell+t_{0}\right)}\right) \ell\|\mathbf{b}\| .
\end{aligned}
$$

\section{STATE OBSERVER DESIGN}

In this work we suppose that the state vector $\mathbf{x}$, the unknown parameters vector $\boldsymbol{\theta}$ and the disturbing input $\mathbf{d}$ in the system (2) are bounded without precise information on their upper bounds.

A s s umption 1. Let for the system (2) $\|\mathbf{x}\|<+\infty$, $|\boldsymbol{\theta}|<+\infty$ and $\mathbf{d} \in \mathcal{M}_{R^{m}}$.

The outline of this section is as follows. In the first part we introduce observer equations and substantiate the observer properties for the case when the exact upper estimates for $\|\mathbf{x}\|,|\boldsymbol{\theta}|$ and $\|\mathbf{d}\|$ are given. In the second part a pro- 
cedure is proposed, which is used for verification of accuracy for the given upper estimates for $\|\mathbf{x}\|,|\boldsymbol{\theta}|$ and $\|\mathbf{d}\|$. In the third part the equations of the proposed hybrid state observer for the system (2) are presented and global convergence to zero of estimation error is proven.

\subsection{Sliding mode state observer}

Consider the following robust state observer for (2):

$$
\dot{\mathbf{z}}=\mathbf{A} \mathbf{z}+\boldsymbol{\varphi}(\mathbf{y})+\mathbf{B}[\mathbf{f}(\mathbf{z}, 0)+\mathbf{u}]+\mathbf{K}(\mathbf{y}-\mathbf{C z}),
$$

where $\mathbf{z} \in R^{n}$ serves as vector $\mathbf{x}$ estimate; $\mathbf{K}$ is observer matrix gain with dimension $n \times p$, which value will be specified later; and $\mathbf{u} \in R^{p}$ is additional sliding mode feedback. Introduce into consideration dynamics of observation error $\mathbf{e}=\mathbf{x}-\mathbf{z}$ :

$$
\dot{\mathbf{e}}=[\mathbf{A}-\mathbf{K C}] \mathbf{e}+\mathbf{B}[\mathbf{f}(\mathbf{x}, \mathbf{d})+\mathbf{G}(\mathbf{x}) \boldsymbol{\theta}-\mathbf{f}(\mathbf{z}, 0)-\mathbf{u}]
$$

and define

$$
\mathbf{u}=\left\{\begin{array}{l}
k|\mathbf{C e}|^{-1} \mathbf{C e} \text { if } \mathbf{C e} \neq 0 ; \\
0 \text { otherwise, }
\end{array}\right.
$$

where $k>0$ is design parameter to be calculated later.

From lemma 1 the following inequalities are satisfied for all $\mathbf{x} \in R^{n}, \mathbf{z} \in R^{n}, \mathbf{d} \in R^{m}$ :

$$
\begin{aligned}
& |\mathbf{f}(\mathbf{x}, \mathbf{d})-\mathbf{f}(\mathbf{z}, 0)| \leq \alpha_{1}(1+|(\mathbf{x}, \mathbf{d})|) \times \\
& \times \alpha_{2}(1+|\mathbf{z}|)|(\mathbf{x}, \mathbf{d})-(\mathbf{z}, 0)|,|\mathbf{G}(\mathbf{x})| \leq \sigma(1+|\mathbf{x}|)
\end{aligned}
$$

for some functions $\alpha_{1}, \alpha_{2}, \sigma \in K$. For $\mathbf{d} \in \mathcal{M}_{R^{m}}$ the last inequality can be rewritten as follows:

$$
\begin{aligned}
& \alpha_{1}(1+|(\mathbf{x}, \mathbf{d})|) \alpha_{2}(1+|\mathbf{z}|)|(\mathbf{x}, \mathbf{d})-(\mathbf{z}, 0)| \leq \\
& \leq \alpha_{1}(1+|\mathbf{x}|+\|\mathbf{d}\|) \alpha_{2}(1+|\mathbf{z}|)[|\mathbf{x}-\mathbf{z}|+\|\mathbf{d}\|] .
\end{aligned}
$$

Denote

$$
\begin{gathered}
L(X, D, Z)=\alpha_{1}(1+X+D) \alpha_{2}(1+Z), \\
F(X, D, Z)=\alpha_{1}(1+X+D) \alpha_{2}(1+Z)[X+Z+D],
\end{gathered}
$$

then for all $|\mathbf{x}| \leq X,|\mathbf{z}| \leq Z,|\boldsymbol{\theta}| \leq \theta$ and $\|\mathbf{d}\| \leq D$,

$|\mathbf{f}(\mathbf{x}, \mathbf{d})-\mathbf{f}(\mathbf{z}, 0)| \leq L(X, D, Z)[|\mathbf{x}-\mathbf{z}|+D]$,

$|\mathbf{f}(\mathbf{x}, \mathbf{d})-\mathbf{f}(\mathbf{z}, 0)| \leq F(X, D, Z),|\mathbf{G}(\mathbf{x}) \boldsymbol{\theta}| \leq \sigma(1+X) \theta$.

As in conventional sliding mode observer approach [7], [8], [31], [37] the following property is required.

A s sumption 2. The system (2) (the matrices A, B and $\mathbf{C})$ is hyper minimum phase.

Under this assumption according to lemma 2 there exist positive definite symmetric matrices $\mathbf{P}$ and $\mathbf{Q}$ such, that

$$
\mathbf{P}(\mathbf{A}-\mathbf{K C})+(\mathbf{A}-\mathbf{K C})^{T} \mathbf{P}=-\mathbf{Q}, \mathbf{P B}=\mathbf{C}^{T} .
$$

Consider for the system (4), (5) Lyapunov function $V(\mathbf{e})=\mathbf{e}^{T} \mathbf{P e}$, which time derivative has form:

$$
\begin{aligned}
& \dot{V}=\mathbf{e}^{T}\left[(\mathbf{A}-\mathbf{K C})^{T} \mathbf{P}+\mathbf{P}(\mathbf{A}-\mathbf{K} \mathbf{C})\right] \mathbf{e}+ \\
& +2 \mathbf{e}^{T} \mathbf{P} \mathbf{B}[\mathbf{f}(\mathbf{x}, \mathbf{d})+\mathbf{G}(\mathbf{x}) \boldsymbol{\theta}-\mathbf{f}(\mathbf{z}, 0)-\mathbf{u}]=-\mathbf{e}^{T} \mathbf{Q} \mathbf{e}+ \\
& +2 \mathbf{e}^{T} \mathbf{C}^{T}[\mathbf{f}(\mathbf{x}, \mathbf{d})+\mathbf{G}(\mathbf{x}) \boldsymbol{\theta}-\mathbf{f}(\mathbf{z}, 0)-\mathbf{u}] .
\end{aligned}
$$

Assume that $\|\mathbf{x}\| \leq X_{0},\|\mathbf{d}\| \leq D_{0},|\boldsymbol{\theta}| \leq \theta_{0}$ and $\|\mathbf{z}\| \leq Z_{0}$ for some $X_{0}>0, D_{0}>0, \theta_{0}>0, Z_{0}>0$, i.e. additionally to assumption 1 suppose that upper estimates on the system (2) state and disturbances are known (constant $Z_{0}$ is always assigned by a designer), then we obtain:

$$
\dot{V} \leq-\mathbf{e}^{T} \mathbf{Q e}+2|\mathbf{C e}|\left[F\left(X_{0}, D_{0}, Z_{0}\right)+\sigma\left(1+X_{0}\right) \theta_{0}-k\right] .
$$

In this case $k>F\left(X_{0}, D_{0}, Z_{0}\right)+\sigma\left(1+X_{0}\right) \theta_{0}$ is a natural choice, then

$$
\dot{V}(t) \leq-2 \alpha V(t), \alpha=0.5 \lambda_{\min }(\mathbf{Q}) / \lambda_{\max }(\mathbf{P}),
$$

where $\lambda_{\min }(\mathbf{Q}), \lambda_{\max }(\mathbf{P})$ are minimum and maximum eigenvalues of corresponding matrices, $t \geq t_{0} \geq 0$. Thus, observation error $\mathbf{e}$ admits the exponential estimate $\left(\left|\mathbf{e}\left(t_{0}\right)\right| \leq\left|\mathbf{z}\left(t_{0}\right)\right|+X_{0}, \rho=\sqrt{\lambda_{\max }(\mathbf{P}) / \lambda_{\min }(\mathbf{P})}\right)$ :

$$
|\mathbf{e}(t)| \leq \rho\left(X_{0}+Z_{0}\right) e^{-\alpha\left(t-t_{0}\right)}, t \geq t_{0} .
$$

\subsection{Observer gains failures verification}

Unfortunately the values $X_{0}, D_{0}, \theta_{0}$ are not known and in general case for particular $k$ the inequality $k>F\left(X_{0}, D_{0}, Z_{0}\right)+\sigma\left(1+X_{0}\right) \theta_{0}$ can be violated. Therefore, it is necessary to propose a procedure for values $X_{0}$, $D_{0}, \theta_{0}$ validation. To do so, denote $\mathbf{e}_{y}=\mathbf{C e}$ and consider dynamics of this variable:

$$
\begin{aligned}
\dot{\mathbf{e}}_{y}= & \mathbf{C}[\mathbf{A}-\mathbf{K C}] \mathbf{e}+\mathbf{C} \mathbf{B}[\mathbf{f}(\mathbf{x}, \mathbf{d})+\mathbf{G}(\mathbf{x}) \boldsymbol{\theta}- \\
& \left.-\mathbf{f}(\mathbf{z}, 0)-k\left|\mathbf{e}_{y}\right|^{-1} \mathbf{e}_{y}\right] .
\end{aligned}
$$

Time derivative of auxiliary Lyapunov function $W\left(\mathbf{e}_{y}\right)=0.5 \mathbf{e}_{y}^{T} \mathbf{e}_{y}$ takes form:

$$
\begin{aligned}
\dot{W} & =\mathbf{e}_{y}^{T} \mathbf{C}[\mathbf{A}-\mathbf{K C}] \mathbf{e}-k \mathbf{e}_{y}^{T} \mathbf{C B}\left|\mathbf{e}_{y}\right|^{-1} \mathbf{e}_{y}+ \\
& +\mathbf{e}_{y}^{T} \mathbf{C B}[\mathbf{f}(\mathbf{x}, \mathbf{d})+\mathbf{G}(\mathbf{x}) \boldsymbol{\theta}-\mathbf{f}(\mathbf{z}, 0)] .
\end{aligned}
$$

If constants $X_{0}, D_{0}, \theta_{0}, Z_{0}$ are chosen high enough, the properties $\|\mathbf{x}\| \leq X_{0},\|\mathbf{d}\| \leq D_{0},|\boldsymbol{\theta}| \leq \theta_{0},\|\mathbf{z}\| \leq Z_{0}$ are true and $k \mathbf{e}_{y}^{T} \mathbf{C B}\left|\mathbf{e}_{y}\right|^{-1} \mathbf{e}_{y} \geq k \lambda_{\min }(\mathbf{C B})\left|\mathbf{e}_{y}\right|$, then

$$
\begin{aligned}
\dot{W} & \leq\left|\mathbf{e}_{y}\right|\{|\mathbf{C}[\mathbf{A}-\mathbf{K C}] \| \mathbf{e}|+|\mathbf{C B}| \times \\
& \times\left[L\left(X_{0}, D_{0}, Z_{0}\right)|\mathbf{e}|+\sigma\left(1+X_{0}\right) \theta_{0}+D_{0}\right]- \\
& \left.-k \lambda_{\min }(\mathbf{C B})\right\} .
\end{aligned}
$$

If $k>F\left(X_{0}, D_{0}, Z_{0}\right)+\sigma\left(1+X_{0}\right) \theta_{0}$ and the estimate (6) is satisfied, then for any $\varepsilon>0$ there exists time $T_{\varepsilon}\left(X_{0}, Z_{0}\right) \geq t_{0}$ such that $|\mathbf{e}(t)| \leq \varepsilon$ for $t \geq T_{\varepsilon}\left(X_{0}, Z_{0}\right)$, where

$$
T_{\varepsilon}(X, Z)=t_{0}-\alpha^{-1} \ln (\varepsilon /\{\rho(X+Z)\}) .
$$

Time $T_{\varepsilon}\left(X_{0}, Z_{0}\right)$ defines length of the time interval, which is required for system (3) to observe state $\mathbf{x}$ of system (2) with predefined accuracy $\varepsilon$. Let additionally

$$
\begin{aligned}
k \geq & \lambda_{\min }(\mathbf{C B})^{-1}\{1+|\mathbf{C}[\mathbf{A}-\mathbf{K} \mathbf{C}]| \varepsilon+ \\
& \left.+|\mathbf{C B}|\left[L\left(X_{0}, D_{0}, Z_{0}\right) \varepsilon+\sigma\left(1+X_{0}\right) \theta_{0}+D_{0}\right]\right\},
\end{aligned}
$$


then for $t \geq T_{\mathcal{E}}\left(X_{0}, Z_{0}\right)$,

$$
\dot{W}(t) \leq-\left|\mathbf{e}_{y}\right|=-\sqrt{2} \sqrt{W(t)}
$$

and for $T_{\varepsilon}\left(X_{0}, Z_{0}\right) \leq t \leq T_{0}\left(X_{0}, Z_{0}\right)$

$$
\begin{gathered}
W(t) \leq 0.5\left(\left|\mathbf{e}_{y}\left(T_{\varepsilon}\left(X_{0}, Z_{0}\right)\right)\right|+T_{\varepsilon}\left(X_{0}, Z_{0}\right)-t\right)^{2} ; \\
W(t)=0, t \geq T_{0}\left(X_{0}, Z_{0}\right),
\end{gathered}
$$

where $\quad T_{0}\left(X_{0}, Z_{0}\right)=\left|\mathbf{e}_{y}\left(T_{\varepsilon}\left(X_{0}, Z_{0}\right)\right)\right|+T_{\varepsilon}\left(X_{0}, Z_{0}\right)$.

Thus, the time $T_{0}\left(X_{0}, Z_{0}\right)$ can be used for detection of correctness of values $X_{0}, D_{0}, \theta_{0}, Z_{0}$ choice, since available for measurements signal $\mathbf{e}_{y}(t)=\mathbf{y}(t)-\mathbf{C z}(t)$ should possess the constrain $\mathbf{e}_{y}(t)=0$ for $t \geq T_{0}\left(X_{0}, Z_{0}\right)$.

\subsection{Global robust hybrid observer for locally Lipschitz} nonlinear systems

Assume that there exists $t^{\prime} \geq T_{0}\left(X_{0}, Z_{0}\right)$ such, that $\left|\mathbf{e}_{y}\left(t^{\prime}\right)\right|>0$, then it means that constants $X_{0}, D_{0}, Z_{0}$ have been chosen not sufficiently high. Taking for $X_{0}, D_{0}$, $\theta_{0}$ and $Z_{0}$ new higher values it is necessary to repeat all described above steps, which in general case can be formalized as follows:

$$
\begin{aligned}
& X_{i}=h_{x}\left(i, X_{i-1}\right), D_{i}=h_{d}\left(i, D_{i-1}\right), \theta_{i}=h_{\theta}\left(i, \theta_{i-1}\right),(7) \\
& Z_{i}=h_{z}\left(i, Z_{i-1}\right), X_{0}>0, D_{0}>0, \theta_{0}>0, Z_{0}>|\mathbf{z}(0)| \text {, } \\
& i=1,2,3, \ldots N \leq+\infty \text {; } \\
& k_{i}>\max \left\{F\left(X_{i}, D_{i}, Z_{i}\right)+\sigma\left(1+X_{i}\right) \theta_{i},\{1+\right. \\
& +|\mathbf{C}[\mathbf{A}-\mathbf{K C}]| \varepsilon+|\mathbf{C B}|\left[L\left(X_{i}, D_{i}, Z_{i}\right) \varepsilon+\right. \\
& \left.\left.\left.+\sigma\left(1+X_{i}\right) \theta_{i}+D_{i}\right]\right\} / \lambda_{\min }(\mathbf{C B})\right\} ; \\
& \dot{\mathbf{z}}(t)=\operatorname{Proj}\left[\mathbf{z}, \mathbf{A} \mathbf{z}(t)+\boldsymbol{\varphi}(\mathbf{y}(t))+\mathbf{B} \mathbf{u}_{i}(t)+\right. \\
& +\mathbf{B} \mathbf{f}(\mathbf{z}(t), 0)+\mathbf{K}(\mathbf{y}(t)-\mathbf{C z}(t))], \\
& \mathbf{u}_{i}(t)=k_{i}|\mathbf{y}(t)-\mathbf{C} \mathbf{z}(t)|^{-1}[\mathbf{y}(t)-\mathbf{C} \mathbf{z}(t)] ;
\end{aligned}
$$

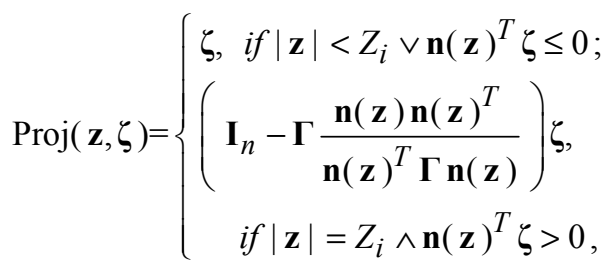

$$
\begin{aligned}
& t_{i+1}=\underset{t \geq T_{i}^{0}}{\arg \sup }\left\{\left|\mathbf{e}_{y}(t)\right|>0\right\}, t_{0}=0, \\
& T_{i}^{0}=\left|\mathbf{e}_{y}\left(T_{i}^{\varepsilon}\right)\right|+T_{i}^{\varepsilon}, T_{i}^{\varepsilon}=t_{i}-\frac{1}{\alpha} \ln \left(\frac{\varepsilon}{\rho\left(X_{i}+Z_{i}\right)}\right),
\end{aligned}
$$

where discrete systems (7) have well defined strictly increasing solutions for any $X_{0}>0, D_{0}>0, \theta_{0}>0, Z_{0}>0$ for all $i \geq 1$, constant $\varepsilon>0$ and matrix $\Gamma>0$ can be taken arbitrary; $\mathbf{n}(\mathbf{z})$ is the unit outward normal vector for $|\mathbf{z}|=Z_{i}$, then projection algorithm (10) ensures existence and boundedness of the system (9) solutions [17], [26] for cases of wrong choices of values $X_{i}, D_{i}, \theta_{i}, Z_{i}$.
The following result describes stability properties of this hybrid observation algorithm.

The or e m 1. Let assumptions 1, 2 hold and discrete systems (7) have well defined strictly increasing to infinity solutions for any $X_{0}>0, D_{0}>0, \theta_{0}>0, Z_{0}>0$ for all $i \geq 1$. Then for any $\varepsilon>0, \Gamma>0$ for the system (2) with the algorithm (7)-(12) it holds that

$-\|\mathbf{z}\|<+\infty$;

- there exists the last step $N<+\infty$ of the algorithm such, that $|\mathbf{y}(t)-\mathbf{C z}(t)|=0$ for all $t \geq T_{N}^{0}$;

- there exists $T_{N}^{\varepsilon} \leq T<+\infty$ such that

$$
|\mathbf{x}(t)-\mathbf{z}(t)| \leq \varepsilon \text { for all } t \geq T \text {. }
$$

According to the result of Theorem 1 the observer (7)-(12) provides finite time convergence of observation error to $\varepsilon$-neighborhood of zero for any $\varepsilon>0$ for all initial conditions $\mathbf{x}_{0} \in R^{n}$ and $\mathbf{d} \in \mathcal{M}_{R^{m}}$ when the corresponding solutions are bounded (assumption 1 is satisfied). The main restrictions on class of admissible for the approach systems (2) are formulated in assumption 2: the linear part of the system (2) has to be hyper minimum phase, that, for instance, implies relative degree 1 condition.

Remark 1. Let us stress that application of adaptive control approach for continuous tuning of the gain $k$ in sense of works [18], [39] is not possible here in general case. Indeed, the desired value for the gain $k$ can be defined by function $F(\|\mathbf{x}\|,\|\mathbf{d}\|,\|\mathbf{z}\|)$, where $\|\mathbf{x}\|$ and $\|\mathbf{d}\|$ are unknown constant, while $\|\mathbf{z}\|$ is the state of observer. Thus, the desired value $F(\|\mathbf{x}\|,\|\mathbf{d}\|,\|\mathbf{z}\|)$ is not a constant and depends in nonlinear fashion on $\mathbf{z}$. This obstruction comes from lemma 1 (Lipschitz constant of locally Lipschitz system nonlinearly depends on all arguments).

To resolve this "loop" logic-based scheme is used and discrete algorithms (7) are applied in this work.

R e mark 2. Optimization of functions $h_{x}, h_{d}, h_{z}, h_{\theta}$ form can guarantee convergence of the algorithm with minimum number of steps or at least it can provide a desired upper estimate on number of the algorithm steps. For example, let $h_{x}\left(i, X_{i}\right)=\exp (\gamma i)$ for some $\gamma>0$, then $N=\operatorname{round}\left\{\gamma^{-1} \ln (\|\mathbf{x}\|)\right\}$, where round $\{\cdot\}$ is rounding-off operator to closest bigger integer number.

\section{ADAPTIVE OBSERVER DESIGN}

Let us consider the following adaptive observer for the system (2):

$$
\dot{\zeta}=\mathbf{A} \zeta+\varphi(\mathbf{y})+\mathbf{B f}(\mathbf{z}, 0)+\mathbf{K}(\mathbf{y}-\mathbf{C} \zeta),
$$

where $\zeta \in R^{n}$ is a new estimate for the vector $\mathbf{x}$, it has form similar to (3). The equation for dynamics of observation error $\boldsymbol{\varepsilon}=\mathbf{x}-\boldsymbol{\zeta}$ can be written as follows: 


$$
\begin{aligned}
\dot{\boldsymbol{\varepsilon}} & =[\mathbf{A}-\mathbf{K C}] \boldsymbol{\varepsilon}+\mathbf{B}[\mathbf{f}(\mathbf{x}, \mathbf{d})-\mathbf{f}(\mathbf{z}, 0)+\mathbf{G}(\mathbf{x}) \boldsymbol{\theta}]= \\
& =[\mathbf{A}-\mathbf{K C}] \boldsymbol{\varepsilon}+\mathbf{B}[\boldsymbol{\eta}+\mathbf{G}(\mathbf{z}) \boldsymbol{\theta}],
\end{aligned}
$$

where $\boldsymbol{\eta}=\mathbf{f}(\mathbf{x}, \mathbf{d})-\mathbf{f}(\mathbf{z}, 0)+\{\mathbf{G}(\mathbf{x})-\mathbf{G}(\mathbf{z})\} \boldsymbol{\theta}$ is a bounded signal (under conditions of theorem 1) and according to lemma 1

$$
\begin{aligned}
|\boldsymbol{\eta}| \leq & |\mathbf{f}(\mathbf{x}, \mathbf{d})-\mathbf{f}(\mathbf{z}, 0)|+|\mathbf{G}(\mathbf{x})-\mathbf{G}(\mathbf{z}) \| \mathbf{\theta}| \leq \\
\leq & \alpha_{1}(1+|\mathbf{x}|+\|\mathbf{d}\|) \alpha_{2}(1+|\mathbf{z}|)[|\mathbf{e}|+\|\mathbf{d}\|]+ \\
& +\beta_{1}(1+|\mathbf{x}|) \beta_{2}(1+|\mathbf{z}|)|\mathbf{e} \| \mathbf{\theta}|,
\end{aligned}
$$

where $\alpha_{1}, \alpha_{2}, \beta_{1}, \beta_{2} \in K$.

Let $\hat{\boldsymbol{\theta}} \in R^{p}$ be an estimate of the uncertain vector $\boldsymbol{\theta}$. To design adaptation algorithm for $\hat{\boldsymbol{\theta}}$ let us introduce into consideration two auxiliary variables $\boldsymbol{\Omega} \in R^{n \times p}$ and $\boldsymbol{\delta}=\boldsymbol{\varepsilon}-\boldsymbol{\Omega} \boldsymbol{\theta}$, which dynamics are defined as follows:

$$
\begin{gathered}
\dot{\boldsymbol{\Omega}}=(\mathbf{A}-\mathbf{K C}) \boldsymbol{\Omega}+\mathbf{B} \mathbf{G}(\mathbf{z}), \\
\dot{\boldsymbol{\delta}}=[\mathbf{A}-\mathbf{K C}] \boldsymbol{\delta}+\mathbf{B} \boldsymbol{\eta} .
\end{gathered}
$$

Under assumptions 1,2 both this variables are bounded (the matrix $\mathbf{A}-\mathbf{K C}$ is Hurwitz). Adaptation algorithm takes form

$$
\dot{\hat{\boldsymbol{\theta}}}=\gamma \boldsymbol{\Omega}^{T} \mathbf{C}^{T}([\mathbf{y}-\mathbf{C} \boldsymbol{\zeta}]-\mathbf{C} \boldsymbol{\Omega} \widehat{\boldsymbol{\theta}}), \gamma>0 .
$$

The or e $\mathrm{m} 2$. Let all conditions of theorem 1 hold and signal $\mathbf{C} \boldsymbol{\Omega}(t)$ be $(\ell, \vartheta)-P E$ for some $\ell>0, \vartheta>0$. Then additionally for the system (13)-(15) for any $\gamma>0$

$$
\begin{aligned}
& -\|\boldsymbol{\zeta}\|<+\infty,\|\boldsymbol{\Omega}\|<+\infty,\|\hat{\boldsymbol{\theta}}\|<+\infty ; \\
& \text { - the following asymptotic estimate holds } \\
& \lim _{t \rightarrow+\infty}|\boldsymbol{\theta}-\hat{\boldsymbol{\theta}}(t)| \leq\left(1+2 \vartheta^{-1} \gamma^{-1} e^{-0.5 \vartheta \gamma}\right) \ell \gamma\left[\rho|\mathbf{C} \| \mathbf{B}| \alpha^{-1}\right]^{2} \times \\
& \times \mathrm{H}(\|\mathbf{x}\|,\|\mathbf{d}\|,|\boldsymbol{\theta}|, \varepsilon) \sigma(1+\|\mathbf{x}\|+\varepsilon), \\
& \mathrm{H}(X, D, \theta, \varepsilon)=\alpha_{1}(1+X+D) \alpha_{2}(1+X+\varepsilon)[\varepsilon+D]+ \\
& +\beta_{1}(1+X) \beta_{2}(1+X+\varepsilon) \varepsilon \theta \text {. }
\end{aligned}
$$

In accordance with the second statement of the theorem the proposed identification algorithm (consisting in observer (13), auxiliary filter (14) and adaptation algorithm (15)) estimates the value $\boldsymbol{\theta}$ with accuracy proportional to $\varepsilon$ for the case of disturbances absence.

R e mark 3 . It is necessary to introduce additional observer (13) instead of (9) enlarging dimension of the final observer (7)-(15) due to special properties of errors $\mathbf{y}-\mathbf{C z}$ and $\mathbf{y}-\mathbf{C} \boldsymbol{\zeta}$. The first one becomes identically zero for all $t \geq T$ that eliminates identification abilities of the algorithm (15), therefore the signal $\mathbf{y}-\mathbf{C} \zeta$ is used in (15).

\section{Simulations}

Let us consider a second order mechanical oscillator [40]:

$$
\begin{gathered}
\mathbf{A}=[-12 ; 11], \mathbf{B}=[0 ; 1]^{T}, \mathbf{C}=\mathbf{B}^{T}, \\
\varphi(y)=0, f(\mathbf{x}, d)=-x_{1}^{3}+d, G(\mathbf{x})=x_{1}^{2}, \theta=-1,
\end{gathered}
$$

where matrix $\mathbf{A}$ is unstable, but the system has globally bounded solutions and it is oscillating (assumption 1 holds).
Since $\varphi(s)=s+1$ and $\lim _{s \rightarrow+\infty} s W(s)=1$, the system is hyper minimum phase and assumption 2 is satisfied. According to theorem 1 the observer (7)-(12) has to ensure robust state estimation for any initial conditions and bounded disturbances. From theorem 2 the identification algorithm (13)-(15) should estimate value $\theta$. For $\mathbf{K}=[2 ; 2]^{T}$ we have $\mathbf{P}=\mathbf{I}_{2}$ and $\mathbf{Q}=[-21 ; 1-2]$, $\alpha=\rho=1 . \quad$ Let $\quad k_{0}=1, \quad d(t)=\sin (0.1 t)$, $X_{0}=D_{0}=\theta_{0}=Z_{0}=0.1, \varepsilon=0.1, \gamma=1$, then for initial conditions $\mathbf{x}(0)=[1 ; 1]$ and $\mathbf{x}(0)=[2 ; 2]$ (the rest initial values are taken zeros) the corresponding graphics of the system (16) output are presented in Fig. 1, norms of observer error $\mathbf{e}$ are plotted in Fig. 2, increasing gains of the observer $k_{i}$ are shown in Fig 3 (the former two in logarithmic scales), graphic of $\hat{\theta}(t)$ is plotted in Fig. $4,0 \leq t \leq 200$ sec.

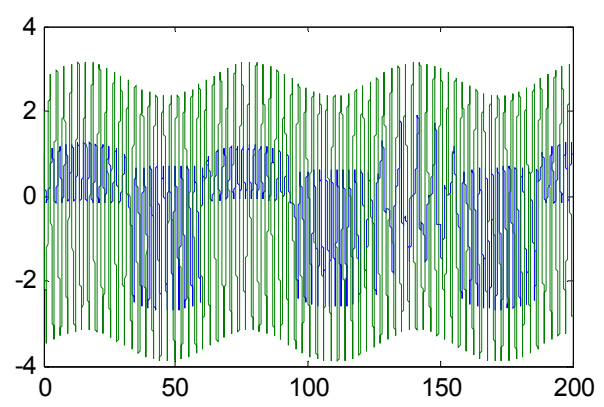

Fig. 1. The system (16) output.

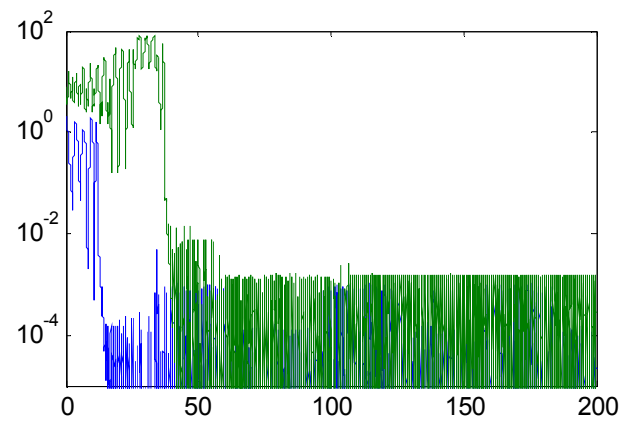

Fig. 2. Norms of observation error $\mathbf{e}$.

According to this simulation the observer gain quickly increases ensuring required quality of observation, while the output then demonstrates complex oscillations changing range and frequency. Asymptotical error fluctuations are proportional to accuracy of the simulation performed in MATLAB 7.0.1.

\section{CONCLUSION}

The procedure for hybrid adaptive observer design for nonlinear locally Lipschitz systems is proposed. Possible presence of signal and parametric uncertainties is taken into account. The solution is based on logic-based control approach applicable to nonlinear systems with bounded solutions. The proposed procedure consists in two steps, first, 
the hybrid robust state observer is designed, then the adaptive one is augmented. Computer simulations confirm applicability and performance of the proposed observer.

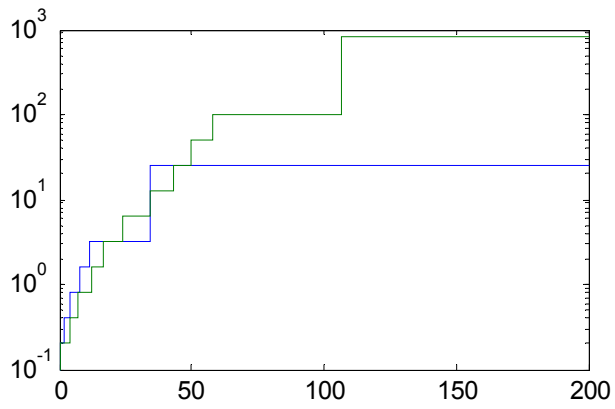

Fig. 3. Observer gains $k_{i}$.

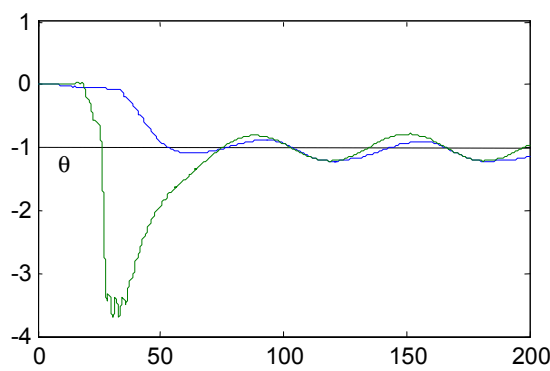

Fig. 4. Adaptation parameter $\hat{\theta}$.

\section{REFERENCES}

[1] Alessandri A. (2004). Observer design for nonlinear systems by using input-to-state stability. Proc. 43rd IEEE Conf. on Decision and Control, December 14-17, Atlantis, Paradise Island, Bahamas, pp. $3892-$ 3897.

[2] Angeli D., Sontag E.D. (1999). Forward completeness, unboundedness observability, and their Lyapunov characterizations. Systems and Control Letters, 38, pp. $209-217$.

[3] Besançon G. (2003). High-gain observation with disturbance attenuation and application to robust fault detection. Automatica, 39, pp. 1095-1102.

[4] Busawon K., Farza M., Hammouri H. (1998). A simple observer for a Class of Nonlinear Systems. Appl. Math. Letters, 11(3), pp. 27-31.

[5] Ciccarella C., Dalla Mora M., Germani A. (1993). A Luenberger-like observer for nonlinear systems. Int. J. Control, 57, pp. 537-556.

[6] Cho Y.M., Rajamani R. (1997) A systematic approach to Adaptive Observer Synthesis for Nonlinear Systems. IEEE Trans. Autom. Control, 42(4), pp. 534-537.

[7] Edwards C., Spurgeon S. K. (1998). Sliding mode control: theory and applications. London: Taylor and Francis Ltd.

[8] Edwards C., Spurgeon S. K., Patton R. J. (2000). Sliding mode observers for fault detection and isolation. Automatica, 36, pp. 541-553.

[9] Deza F., Bossanne D., Busvelle E., Gauthier J. P., Rakotopara D.(1993). Exponential observer for nonlinear systems. IEEE Trans. Autom. Control, 38(3), pp. 482-484.

[10] Efimov D.V., Fradkov A.L. (2006). Adaptive Tuning to Bifurcation for Time-Varying Nonlinear Systems. Automatica, 42, pp. 417-425.

[11] Efimov D.V. (2006). Dynamical adaptive synchronization. International Journal of Adaptive Control and Signal Processing, 20, pp. 491-507.

[12] Fradkov, A.L., Nikiforov V.O., Andrievsky B.R. (2002). Adaptive observers for nonlinear nonpassifiable systems with application to signal transmission. Proc. $41^{\text {th }}$ IEEE Conf. Decision and Control, Las Vegas, $10-13$ Dec., pp. $4706-4711$.

[13] Garg V., Hedrick J. (1996). Fault detection filters for a class of nonlinear systems. Proc. Amer. Control Conf., Seattle, WA, Jun. 1996, pp. $1647-1651$.
[14] Johansson A., Medvedev A. (2003). An observer for systems with nonlinear output map. Automatica, 39(5), pp. 909-918.

[15] Khalil H.K. (2005). A note on the robustness of high-gain-observerbased controllers to unmodeled actuator and sensor dynamics. Automatica, 41, pp. 1821-1824.

[16] Kreisselmeier G., Engel R. (2003). Nonlinear observers for autonomous Lipschitz continuous systems. IEEE Trans. Autom. Control, 48(3), pp. 397-401.

[17] Krstić M., Kanellakopoulos I., Kokotović P.V. (1995). Nonlinear and adaptive control design. New York Wiley.

[18] Lei H., Wei J., Lin W. (2005). A global observer for observable autonomous systems with bounded solution trajectories. Proc. $44 \mathrm{rd}$ IEEE Conf. on Decision and Control, December 12-15, 2005, Sevilla, Spain, pp. $1911-1916$.

[19] Marino R. (1990). Adaptive observers for single-ouput nonlinear systems. IEEE Trans. Autom. Control, 35(9), pp. 1054-1058.

[20] Marino R., Tomei P. (1992). Adaptive observers for a class of multioutput nonlinear systems. Int. J. Adaptive Contr. Signal Processing, $\mathbf{6}$, pp. 353-365.

[21] Nijmeijer H., Fossen T.I. (1999). New Directions in Nonlinear Observer Design. London, U.K.: Springer-Verlag.

[22] Nijmeijer H., Mareels I.M.Y. (1997). An Observer Looks at Synchronization. IEEE Trans. Circuit Syst. I, 44(10), pp. $882-890$.

[23] Nikoukhah R. (1998). A new methodology for observer design and implementation. IEEE Trans. Autom. Control, 43(2), pp. 229-234.

[24] Pecora L. and T. Carrol (1990). Synchronization in chaotic systems, Phys.Rev. Lett., 64, pp. $821-824$.

[25] Pertew A.M., Marquez H.J., Zhao Q. (2006). Hinf Observer Design for Lipschitz Nonlinear Systems. IEEE Trans. Autom. Control, 51(7), pp. 1211-1216.

[26] Pomet J.-B., Praly L. (1992). Adaptive nonlinear regulation: Estimation from the Lyapunov equation. IEEE Trans. Automat. Contr., 37, pp. 729-740.

[27] Raghavan S. (1992). Observers and compensators for nonlinear systems with application to flexible joint robots. Ph.D. dissertation, Univ. California, Berkeley, CA.

[28] Raghavan S., Hedrick J. (1994). Observer design for a class of nonlinear systems. Int. J. Control, 59, pp. 515-528.

[29] Rajamani R. (1993). Observers for nonlinear systems with application to active automotive suspensions. Ph.D. dissertation, Univ. California, Berkeley, CA.

[30] Rajamani R. (1998). Observers for Lipschitz nonlinear systems. IEEE Trans. Autom. Control, 43(3), pp. 397-401.

[31] Tan C.P., F. Crusca, Aldeen M. (2008). Extended results on robust state estimation and fault detection. Automatica, 44, pp. 2027-2033.

[32] Thau F.E. (1973). Observing the state of non-linear dynamic systems. Int. J. Control, 17(3), pp. 471-479.

[33] Wang H., Huang Z.J., Daley S. (1997). On the use of adaptive updating rules for actuator and sensor fault diagnosis. Automatica, 33(2), pp. 217-225.

[34] Xia X., Zeitz M. (1997). On nonlinear continuous observers. Int. $J$ Control, 66(6), pp. 943-954.

[35] Xu A., Zhang Q. (2004). Nonlinear system fault diagnosis based on adaptive estimation. Automatica, 40, pp. 1181-1193.

[36] Xu A., Zhang Q. (2004). Residual Generation for Fault Diagnosis in Linear Time-Varying Systems. IEEE Trans. Autom. Control, 49(5), pp. 767-772.

[37] Yan X.-G., Edwards C. (2007). Nonlinear robust fault reconstruction and estimation using a sliding mode observer. Automatica, 43, pp. 1605-1614.

[38] Zhang, Q. (2002). Adaptive observer for multiple-input-multipleoutput (MIMO) linear time varying systems. IEEE Trans. Autom. Control, 47(3), pp. 525-529.

[39] Zhou J., Lu J., Lü J. (2006). Adaptive Synchronization of an Uncertain Complex Dynamical Network. IEEE Trans. Autom. Control, 51(4), pp. 652-656.

[40] Bobtsov A., Pyrkin A. Experimental research of consecutive compensator approach on basis of mechatronic systems // 6th EUROMECH Nonlinear Dynamics Conference - Saint-Petersburg, 2008. 